\title{
Backscattering off a dynamical impurity in one-dimensional Fermi systems: A perturbative computation
}

\author{
Carlos M. Naón and Mariano J. Salvay \\ Departamento de Física, Facultad de Ciencias Exactas, Universidad Nacional de La Plata, CC 67, 1900 La Plata, Argentina \\ and Instituto de Física La Plata, Consejo Nacional de Investigaciones Científicas y Técnicas, CC 67, 1900 La Plata, Argentina
}

(Received 18 August 2006; revised manuscript received 12 October 2006; published 8 March 2007)

\begin{abstract}
We investigate the problem of backscattering off a time-dependent and spatially extended barrier in a one-dimensional noninteracting electron gas. By performing a perturbative expansion in the backscattering amplitude, we compute the total energy density of the system. We show how the free fermion spectrum and the conductance of the system are affected by the interplay between dynamical and geometrical properties of the impurity.
\end{abstract}

DOI: 10.1103/PhysRevB.75.113102

PACS number(s): 71.10.Pm, 73.40.Gk, 05.30.Fk, 73.63.Nm

The physics of tunneling through time-dependent barriers is a topic of great importance in the subject of correlated quasi-one-dimensional electron transport. A considerable effort has been made over the last few years, focused on the understanding of quantum transport, both experimentally ${ }^{1}$ and theoretically. ${ }^{2}$ Despite the possible technological advances that the control of charge and spin currents could bring up, we are faced with the fundamental question of what we could learn about electron correlations using timedependent potentials as out-of-equilibrium probes. To answer this question, at least partially, more theoretical work is needed to understand the detailed dynamics induced by this type of perturbation.

An interesting observable that characterizes a tunneling process is the energy resolved current $j(\omega)$. In Ref. 3, the relevance of this quantity in the upper region of the spectrum (i.e., above the Fermi energy, $\omega>E_{F}$ ) was emphasized, in connection to correlations in the leads. To leading order in the tunneling amplitude, the energy resolved current $j(\omega)$ is related to a simpler observable, namely, the electron energy distribution function $n(\omega)$, also referred to as the total energy density (TED) in the literature ${ }^{4}$ [for a precise mathematical definition see Eq. (5) below]. This quantity describes the perturbation of the ground state in the leads due to tunneling processes. For a noncorrelated material, for instance, $n(\omega)$ should vanish above the Fermi surface. Any population of the spectrum above the Fermi energy is originated from a combined effect of correlations and multiparticle tunneling, due, on its turn, to out of equilibrium processes. ${ }^{3}$ In Ref. 5, $n(\omega)$ was evaluated in a model of correlated one-dimensional fermions with a time-dependent impurity coupled to the electron density through a forward-scattering coupling. In Ref. 6 this model was analyzed by means of functional bosonization, ${ }^{7}$ focusing, in particular, on the transients produced by turning on the oscillatory impurity strength.

It is important to notice, however, that backscattering effects are expected to be relevant as a rule, in all but rather exceptional experimental settings. ${ }^{8}$ Possible experimental realizations where backscattering will play a central role are one-dimensional (1D) wires in the presence of a timedependent gate voltage and a Hall bar with a constriction. ${ }^{9}$ The problem of backscattering by dynamical impurities is usually a very difficult one. Some models, like the spinless
Luttinger model with a deltalike impurity, can be solved exactly for the specific value of the Luttinger parameter $K=\frac{1}{2} \cdot{ }^{10}$ However, for general strengths of the electron-electron interaction and finite-ranged impurities, there are no available closed analytical solutions (even in the free case). Therefore it is of fundamental importance to develop different strategies in such a context. Recently, ${ }^{11}$ effects of backscattering in a Luttinger liquid due to a time-dependent ultralocalized impurity were studied perturbatively, finding a striking enhancement of the total current for special values of the Luttinger parameters. An alternative, nonperturbative point of view was adopted in Ref. 12, where an adiabatic approximation was invoked in order to get the distortion of the noncorrelated TED due to the backscattering amplitude and the geometry of the impurity, i.e., for noninteracting fermions in the presence of an extended barrier. Since the main results of Ref. 12 (a peak structure of the TED) were obtained in a strong coupling and low frequency regime $(|\omega| \gg \Omega)$, it is certainly desirable to have a quantitative knowledge of the TED for the same problem (noninteracting fermions with a time-dependent barrier) but in the weak coupling regime and for all external frequencies. This is the main motivation for the present work. We study the effects that the backscattering off an extended dynamical impurity of width $a$ and amplitude $g_{b}$, oscillating with frequency $\Omega$, will have on the spectrum of a one-dimensional noninteracting fermion gas. Our results, though obviously valid only for $\frac{g_{b} a}{\hbar v_{F}}$ sufficiently small, are not restricted to small values of the external frequency. We then expect to capture the main features related to the time-dependent nature of the perturbation. We compute the TED up to second order in the backscattering parameter $\frac{g_{b} a}{\hbar v_{F}}$. We also evaluate the change in the conductance $\Delta G$ produced by the time-dependent barrier. We show that, in contrast to the result obtained in Ref. 11 for a Luttinger system with an ultralocalized impurity (when specializing the result to the case of noninteracting electrons), in a system of noncorrelated electrons an extended geometry gives rise to a nontrivial dependence of $\Delta G$ on the frequency of the perturbation. In particular we find that the conductance of the system remains unchanged for high frequencies $\left(\frac{\Omega a}{v_{F}} \gg 1\right)$. Let us stress that we deal with noninteracting electrons throughout this work. Although the more involved Luttinger liquid 
case is, of course, more important, the analytical results we present here will be helpful as a limiting case of the interacting problem.

As the computational starting point, let us consider the following Hamiltonian, which describes the interaction of spinless fermions with an external effective time-dependent potential $V(x, t)$, responsible for backscattering transitions between right and left movers:

$$
H=H_{0}+H_{\text {imp }}
$$

where

$$
H_{0}=i \hbar v_{F} \int d x\left(\psi_{R}^{\dagger} \partial_{x} \psi_{R}-\psi_{L}^{\dagger} \partial_{x} \psi_{L}\right)
$$

and

$$
H_{\mathrm{imp}}=g_{b} \int d x\left(\psi_{R}^{\dagger} \psi_{L}+\psi_{L}^{\dagger} \psi_{R}\right) V(x, t) .
$$

Above, $g_{b}$ is the coupling constant associated to the backward scattering of electrons caused by the presence of a time-dependent harmonic barrier. Let us mention here that the simultaneous presence of both forward and backward scattering by the impurity does not bring about any new effect, at least up to second order in the couplings. In fact, in the absence of impurity-backscattering the TED has been computed exactly in Refs. 5 and 6, showing a sideband structure that reflects the inelastic nature of time-dependent scattering. Since up to second order in the couplings the crossed term that would relate forward and backward contributions vanishes, the combined effect will be a direct superposition of the above-mentioned results and the ones we present here.

Although we have verified that our method works independently of the explicit details of $V(x, t)$, in order to explore the effect of finite range barriers, we consider a square potential profile,

$$
V(x, t)=[\Theta(x+a / 2)-\Theta(x-a / 2)] \cos (\Omega t),
$$

where $a$ is the width of the square potential and $\Omega$ the oscillation frequency.

We are particularly interested in obtaining the TED for the above model. We recall that in the Wigner representation the TED can be written in terms of the fermion correlation function as

$$
n(\omega, X, T)=-i \int_{-\infty}^{\infty} d \tau e^{i \omega \tau} G_{+-}(r=0, X, \tau, T),
$$

where we have introduced the closed time path formalism ${ }^{13}$ in which fermion propagators are time-ordered along the usual Schwinger-Keldysh time contour:

$$
\begin{gathered}
G_{+-}\left(\mathbf{x}, \mathbf{x}^{\prime}\right)=i\left\langle\Psi^{\dagger}\left(\mathbf{x}^{\prime}\right) \Psi(\mathbf{x})\right\rangle, \\
G_{-+}\left(\mathbf{x}, \mathbf{x}^{\prime}\right)=-i\left\langle\Psi(\mathbf{x}) \Psi^{\dagger}\left(\mathbf{x}^{\prime}\right)\right\rangle, \\
G_{++}\left(\mathbf{x}, \mathbf{x}^{\prime}\right)=-i\left\langle T \Psi(\mathbf{x}) \Psi^{\dagger}\left(\mathbf{x}^{\prime}\right)\right\rangle, \\
G_{--}\left(\mathbf{x}, \mathbf{x}^{\prime}\right)=-i\left\langle\tilde{T} \Psi(\mathbf{x}) \Psi^{\dagger}\left(\mathbf{x}^{\prime}\right)\right\rangle,
\end{gathered}
$$

where $T$ and $\widetilde{T}$ denote the time and antitime ordering operations, respectively. Above, $r, \tau$ and $X, T$ are the spatial and temporal relative and center of mass coordinates, respectively.

In realistic systems the frequency $\Omega$ is expected to be quite high so that it is unlikely that the explicit time resolution of the TED would be experimentally accessible. Then, it is natural to consider the time average, over the period of the perturbation:

$$
\bar{n}(\omega, X)=\frac{\Omega}{2 \pi} \int_{0}^{2 \pi / \Omega} d \operatorname{Tn}(\omega, X, T) .
$$

Let us stress that this averaged TED is a purely dynamical quantity, i.e., in general it is not connected to the static case. In particular, as explained in Ref. 12 the static limit $\Omega \rightarrow 0$ cannot be reproduced from this expression (see below).

At this point we calculate the average TED as an expansion up to the first nontrivial order in the dimensionless parameter $g_{b} a / \hbar v_{F}$. As is usual in the context of $1 \mathrm{D}$ fermionic models, we work in the chiral representation, introducing a spinor $\Psi$ with components $\psi_{L}$ and $\psi_{R}$. Computing the corrections to the Green functions $G_{+-}^{R}$ and $G_{+-}^{L}$ due to the timedependent barrier, one readily verifies that the first order contributions vanish. Considering then the second order corrections, inserting the results in Eq. (5), and finally using the definition given in Eq. (7), we obtain the following expressions for the average TEDs for both right and left components $\left[\overline{n_{R}}(\omega, X)=\overline{n_{L}}(\omega,-X)=N(\omega, X)\right]$ :

$$
\begin{aligned}
N(\omega, X)= & \frac{1}{v_{F}}\left[\Theta(-\omega)-[\Theta(X+a / 2)-\Theta(X-a / 2)]\left(\Theta(-\omega) \frac{g_{b}^{2} a^{2}}{2 \hbar^{2} v_{F}^{2}}\left\{F\left[(2 \omega+\Omega) a / v_{F}\right]+F\left[(2 \omega-\Omega) a / v_{F}\right]\right\}\right.\right. \\
& -\Theta(-\omega) \frac{g_{b}^{2}(a / 2-X)^{2}}{2 \hbar^{2} v_{F}^{2}}\left\{F\left[(2 \omega+\Omega)(a / 2-X) / v_{F}\right]+F\left[(2 \omega-\Omega)(a / 2-X) / v_{F}\right]\right\} \\
& \left.-\Theta(-\omega-\Omega) \frac{g_{b}^{2}(a / 2+X)^{2}}{2 \hbar^{2} v_{F}^{2}} F\left[(2 \omega+\Omega)(a / 2+X) / v_{F}\right]-\Theta(-\omega+\Omega) \frac{g_{b}^{2}(a / 2+X)^{2}}{2 \hbar^{2} v_{F}^{2}} F\left[(2 \omega-\Omega)(a / 2+X) / v_{F}\right]\right) \\
& \left.-\Theta(X-a / 2) \frac{g_{b}^{2} a^{2}}{2 \hbar^{2} v_{F}^{2}}\left\{[\Theta(-\omega)-\Theta(-\omega-\Omega)] F\left[(2 \omega+\Omega) a / v_{F}\right]+[\Theta(-\omega)-\Theta(-\omega+\Omega)] F\left[(2 \omega-\Omega) a / v_{F}\right]\right\}\right],
\end{aligned}
$$




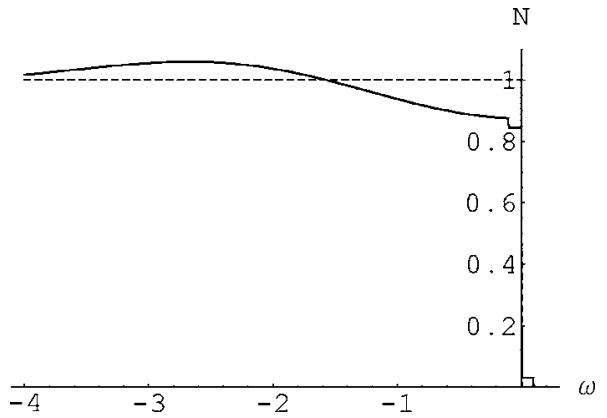

FIG. 1. Averaged TED for $g_{b}=1 / \sqrt{2}, a=1$, and $\Omega=0.1\left(\hbar=v_{F}\right.$ $=1$ ). The dashed line corresponds to the free case.

where $F(z)=\frac{1-\cos z}{z^{2}}$. Let us recall that this result is valid for $\left|\frac{g_{b} a}{\hbar v_{F}}\right| \ll 1$. Analyzing the above expression one sees that the average TED is a superposition of free TEDs centered at the origin of energies and at $\pm \Omega$. This feature is a direct consequence of the perturbative order we are working on. Indeed, we have verified that terms corresponding to larger shifts in $\omega$ will also contribute to $N(\omega)$ when next order corrections are taken into account. In analogy to the behavior of the TED when only forward scattering barriers are present, ${ }^{7}$ the coefficients that weight the contributions of the various free TEDs depend on $\Omega$ and the impurity geometry through the width $a$. However, in contrast to that case, in which the average TED coincides with the static case [which is in turn equal to the free value: $N(\omega)=\Theta(-\omega)]$ at every spatial point when $\Omega \rightarrow 0$, in the present case this limit cannot be obtained inside the barrier. In fact, one gets one-half of the right answer for the static limit in this perturbative order. On the other hand, outside the barrier there is no problem and both results for $N(\omega)$ coincide for $\Omega \rightarrow 0$ [these results are also equal to the free case, see Eq. (8)]. This disagreement found for TEDs evaluated inside the barrier is not surprising since the limit $\Omega \rightarrow 0$ in Eq. (7) is not well-defined.

In Figs. 1 and 2 we display the behavior of $\bar{n}(\omega)=N(\omega)$ at the center of the barrier and for fixed values of $g_{b}$ and $a$. We can identify two different regimes in the behavior of $N(\omega)$, according to the values of $\frac{\Omega a}{v_{F}}$. For $\frac{\Omega a}{v_{F}} \ll 2.67$ (please see Fig. 1), $N(\omega)$ has a maximum in $-\frac{\omega a}{v_{F}} \approx 2.67$; this corresponds to the quasistatic region. Figure 2 shows an example of the other regime, the high-frequency region, where $\frac{\Omega a}{v_{F}} \gg 2.67$. Here we note the appearance of a depression in the spectrum

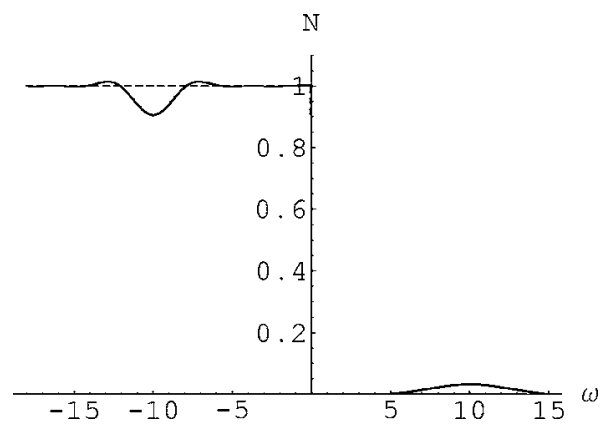

FIG. 2. Averaged TED for $g_{b}=1 / \sqrt{2}, a=1$, and $\Omega=20\left(\hbar=v_{F}\right.$ $=1)$. centered at $\omega=-\Omega / 2$, and a peak at $\omega=\Omega / 2$. The peculiar value $\frac{\Omega a}{v_{F}} \approx 2.67$ has been determined numerically, searching for the region of maximum superposition of both previous effects. It is interesting to note that one peak centered around this value also appears in the context of the adiabatic approximation, when considering the case $g_{b} a=1$ (Figs. 1 and 2 of Ref. 12). However, we do not take the comparison further, since the adiabatic approach of Ref. 12 focused on the strong coupling region, whereas here we deal with the opposite regime.

In contradistinction to the case of forward scattering barriers, impurities of the backscattering type affect the transport properties of the system. In order to obtain the conductance $G$ for this system, we must analyze the linear response of the current under the influence of an external bias $V$. The effect of this voltage can be introduced, as usual, by modifying the Hamiltonian density $\mathcal{H}$ given in Eq. (1) as $\mathcal{H}$ $\rightarrow \mathcal{H}+\mu_{L} \psi_{L}^{\dagger} \psi_{L}$, where $\mu_{L}=-e V$ is the chemical potential coupled to fermions of left chirality. The conductance is then given by

$$
G=\lim _{V \rightarrow 0} \frac{J}{V}
$$

with

$$
J=-i e v_{F} \frac{\Omega}{2 \pi} \int_{0}^{2 \pi / \Omega}\left[G_{+-}^{R}(x, t, x, t)-G_{+-}^{L}(x, t, x, t)\right] d t,
$$

where $J$ is the dc component of the current. As is wellknown, in the absence of impurities, the conductance of a 1D noncorrelated Fermi system is $G=e^{2} / h$, where $h$ is Planck's constant. We have computed the second order correction to $G$ due to the presence of the time-dependent backscattering barrier considered in this work. The result is

$$
\Delta G=-\frac{e^{2}}{h} 2 \frac{g_{b}^{2}}{\hbar^{2}} \frac{\sin ^{2}\left[\Omega a / 2 v_{F}\right]}{\Omega^{2}} .
$$

We then found that the conductance decreases for $\Omega a / 2 v_{F} \neq n \pi$. The magnitude of the effect depends on the amplitude of the barrier, its width, and the frequency of the oscillation. We see that in contrast to the result obtained for a point impurity, ${ }^{11}$ where $\Delta G$ is independent of frequency, we get a nontrivial oscillatory behavior of $\Delta G$ as a function of the external frequency $\Omega$. In particular, in the low frequency regime we predict a maximum decrease of $G$, similar to the behavior corresponding to static barriers, as expected. On the other hand, for $\Omega a / 2 v_{F}=n \pi$ (with $n$ an integer greater than 1) $G$ is not affected by the barrier. The same phenomenon takes place in the high frequency region.

To summarize, we have studied the effect of a backscattering time-dependent barrier on the spectrum and transport properties of a noncorrelated 1D electronic system. We focused our attention not only on the out of equilibrium physics caused by a dynamical impurity but also on the role played by its extended geometry. We performed a perturbative computation of the total energy density of the system 
$N(\omega)$. In the low frequency regime we found a maximum of $N(\omega)$ in $-\frac{\omega a}{v_{F}} \approx 2.67$; this corresponds to the quasistatic region. In the high frequency regime $N(\omega)$ displays a depression in the spectrum centered at $\omega=-\Omega / 2$ and a peak at $\omega$ $=\Omega / 2$. Concerning the conductance of the system we showed that, in contrast to the behavior predicted for an ultralocalized barrier, for an extended impurity it changes as an oscillatory function of $\Omega a / 2 v_{F}$ [see Eq. (11)]. This result, together with the one obtained in Ref. 11 could be used to experimentally characterize the spatial structure of constrictions through conductance measurements.

This work was supported by Universidad Nacional de La Plata and Consejo Nacional de Investigaciones Científicas y Técnicas (CONICET), Argentina.
${ }^{1}$ M. Switkes, C. M. Markus, K. Campman, and A. C. Gossard, Science 293, 1905 (1999); L. DiCarlo, C. M. Marcus, and J. S. Harris, Jr., Phys. Rev. Lett. 91, 246804 (2003).

${ }^{2}$ B. L. Altshuler and L. I. Glazman, Science 283, 1864 (1999); P. W. Brouwer, Phys. Rev. B 58, R10135 (1998); F. Zhou, B. Spivak, and B. Altshuler, Phys. Rev. Lett. 82, 608 (1999); T. A. Shutenko, I. L. Aleiner, and B. L. Altshuler, Phys. Rev. B 61, 10366 (2000); O. Entin-Wohlman, A. Aharony, and Y. Levinson, ibid. 65, 195411 (2002); M. Moskalets and M. Büttiker, ibid. 66, 205320 (2002); 69, 205316 (2004); 70, 245305 (2004); L. Arrachea, ibid. 66, 045315 (2002); 70, 155407 (2004); 72, 125349 (2005); 72, 121306(R) (2005).

${ }^{3}$ A. Komnik and A. O. Gogolin, Phys. Rev. B 66, 035407 (2002).

${ }^{4}$ J. W. Gladzuk and E. W. Palmer, Rev. Mod. Phys. 45, 487 (1973).

${ }^{5}$ A. Komnik and A. O. Gogolin, Phys. Rev. B 66, 125106 (2002).

${ }^{6}$ C. M. Naón, M. J. Salvay, and M. L. Trobo, Phys. Rev. B 70,
195109 (2004)

${ }^{7}$ C. Naón, M. J. Salvay, and M. L. Trobo, Int. J. Mod. Phys. A 19, 4953 (2004).

${ }^{8}$ P. Poncharal, Z. L. Wang, D. Ugarte, and W. A. de Heer, Science 283, 1513 (1999).

${ }^{9}$ F. P. Milliken, C. P. Umbach, and R. A. Webb, Solid State Commun. 97, 309 (1996).

${ }^{10}$ P. Sharma and C. Chamon, Phys. Rev. Lett. 87, 096401 (2001).

${ }^{11}$ D. E. Feldman and Y. Gefen, Phys. Rev. B 67, 115337 (2003).

${ }^{12}$ D. G. Barci, L. Moriconi, M. Moriconi, C. M. Naón, and M. J. Salvay, Phys. Rev. B 72, 235112 (2005).

${ }^{13}$ J. Schwinger, J. Math. Phys. 2, 407 (1961); L. V. Keldysh, Sov. Phys. JETP 20, 1018 (1965); K. Chou, Z. Su, B. Hao, and L. Yu, Phys. Rep. 118, 1 (1985); N. P. Landsman and Ch. G. van Weert, ibid. 145, 141 (1987); A. Das, Finite Temperature Field Theory (World Scientific, Singapore, 1997). 